\title{
The CASA quantitative precipitation estimation system: a five year validation study
}

\author{
V. Chandrasekar, Y. Wang, and H. Chen \\ Colorado State University, 1373 Campus Delivery, Fort Collins, CO 80523, USA \\ Correspondence to: V. Chandrasekar (chandra@engr.colostate.edu)
}

Received: 27 February 2012 - Revised: 13 June 2012 - Accepted: 20 June 2012 - Published: 12 September 2012

\begin{abstract}
Flooding is one of the most common natural hazards that produce substantial loss of life and property. The QPE products that are derived at high spatiotemporal resolution, which is enabled by the deployment of a dense radar network, have the potential to improve the prediction of flash-flooding threats when coupled with hydrological models. The US National Science Foundation Engineering Research Center for Collaborative Adaptive Sensing of the Atmosphere (CASA) is dedicated to revolutionizing our ability to observe, understand, predict, and respond to hazardous weather events, especially in the lower atmosphere. CASA's technology enables precipitation observation close to the ground and QPE is one of the important products generated by the system. This paper describes the CASA QPE system built on the various underlying technologies of networked X-band radar systems providing high-resolution (in space and time) measurements, using the rainfall products from the radar. Evaluation of the networked rainfall product using $5 \mathrm{yr}$ of data from the CASA IP- 1 test bed is presented. Cross validation of the product using $5 \mathrm{yr}$ of data with a gauge network is also provided. The validation shows the excellent performance of the CASA QPE system with a standard error of $25 \%$ and a low bias of $3.7 \%$. Examples of various CASA rainfall products including instantaneous and hourly rainfall accumulations are shown.
\end{abstract}

\section{Introduction}

Flooding is one of the most common natural hazards that produce substantial loss of life and property. According to the US National Academy report, floods are responsible for more deaths nationwide than any other weather phenomenon (NRC, 2005). Especially heavy development in urban regions decreases the response time of urban watersheds to rainfall and increases the chance of localized flooding events over a small spatial domain (Maki et al., 2008). The scales of urban floods are fairly small and intense and have a large temporal variability with fast response time. Successful monitoring of urban floods requires high spatiotemporal resolution and accurate precipitation estimation because of the rapid flood response as well as the complex hydrologic and hydraulic characteristics in an urban environment.

The CASA (Collaborative Adaptive Sensing of the Atmosphere) is a US National Science Foundation Engineering Research Center dedicated to revolutionizing our ability to observe, understand, predict, and respond to hazardous weather events, especially in the lower atmosphere. This center has pursued an innovative, densely networked, sensing paradigm, called DCAS (Distributed Collaborative Adaptive Sensing), to overcome the resolution and coverage limitations of traditional weather radars using lower cost, densely networked radar systems (McLaughlin et al., 2005). The DCAS radar network is capable of high-resolution observation over a large coverage area (Chandrasekar et al., 2008). The network centric sensing paradigm greatly improves the system capability and measurement accuracy (Junyent and Chandrasekar, 2009). By reducing the maximum range and operating at X-band, one can ensure good cross-range resolution with a small-size antenna and keep the radar beam closer to the ground. The individual sensing node can be constructed from small-size radar units that are easily amenable to ubiquitous urban deployment. A recent National Academy study has explained the salient features of the DCAS system for complex terrain (NRC, 2008).

CASA has developed the technologies and the systems for network centric weather observation. A four-node 
X-band dual-polarization radar network has been in operation in Oklahoma, which is referred as the IP1 (Integrative Project 1). Dual polarization radar-based technologies have been implemented for real-time mitigation of rain attenuation. Also the rainfall estimation using specific differential propagation phase (Bringi and Chandrasekar, 2001), $K_{\mathrm{dp}}$, in rain is more sensitive at $\mathrm{X}$-band compared to lower frequencies such as $\mathrm{S}$ - or C-band, as long as the radar return signals are not attenuated fully in rain. Use of $K_{\mathrm{dp}}$ for producing rainfall products makes it immune to absolute calibration errors and also more accurate than the tradition reflectivityrainfall relations (Bringi and Chandrasekar, 2001). One of the major challenges of using $K_{\mathrm{dp}}$ is that it is derived as the slope of a phase quantity. This poses two challenges: namely, the range resolution of a slope is not as good as the point measurements, and the slope estimation is highly sensitive to phase noise. Wang and Chandrasekar (2010) have developed a robust $K_{\mathrm{dp}}$ estimation procedure that overcomes these problems and has contributed significantly to the progress of $K_{\text {dp }}$ estimation at X-band frequencies.

This paper describes the CASA Quantitative Precipitation Estimation (QPE) system built on the various underlying technologies of networked X-band radar systems providing high-resolution (in space and time) measurements, using the rainfall products from the radar. Evaluation of the networked rainfall product using $5 \mathrm{yr}$ of data from the CASA IP- 1 test bed is presented. Cross validation of the product using $5 \mathrm{yr}$ of data with a gauge network is also provided. Examples of various CASA rainfall products including instantaneous and hourly rainfall accumulations are shown. The paper is organized as follows. Section 2 describes the features of the networked radar system for rainfall estimation, whereas Sect. 3 describes the CASA X-band radar network. The algorithmic aspects of the dual polarization radar rainfall estimation are described in Sect. 4, and the architecture and the communication aspects of the CASA QPE system are described in Sect. 5. Section 6 presents samples of the QPE system products and cross validation with gauge network. Section 7 summarizes the important features of the CASA QPE system.

\section{Rainfall mapping using dense radar network}

QPE is one of the key products that will drive the hydrologic models for flood monitoring. High-resolution rainfall mapping over a large area with frequent update is the fundamental strength of radar in this type of applications. In urban environments, the replacement of vegetation by impermeable surface and the construction of drainage networks reduce the response time of urban watersheds to rainfall but also increase the peak discharge volumes. Road, channels, buildings and other structures modify the hydrologic and hydraulic characteristics and potentially increase the chance of localized flooding events. Predication of lumped discharge from the target basin is not sufficient in this case for efficient flood warning and prevention.

The spatial resolution of the QPE depends on the radar parameters such as cross-range resolution, and the temporal resolution depends on the scan strategy. The CASA system of radars maintains a system heartbeat of $30 \mathrm{~s}$, and after finishing two low level full 360 degree scans, conducts adaptive scans at different elevations depending on the location of the echo. The accuracy of radar QPE relies both on the radar system, such as beamwidth and sidelobe, and on the environmental factors, such as clutter and storm variability. Radar beam can overshoot the lower part of atmosphere due to the Earth's curvature or blockage due to local terrain. Radar observations of rainfall can also be contaminated by hail, melting hydrometeor particles, and/or ground clutter. In addition, in urban environments, strong clutter is expected due to scattering from buildings in the neighborhood, which act as perfect reflectors when radar is steered down low. In addition, radar calibration errors and anomalous propagation also contaminate rainfall rate estimation. We refer to Saltikoff et al. (2010), for a summary of the factors leading to the quality assurance of weather radar data for QPE. In this research, the main parameter used for rainfall estimation is specific differential propagation phase $\left(K_{\mathrm{dp}}\right)$.

\subsection{Spatial resolution}

For urban flood monitoring, a high-resolution hydrologic model has been developed on the order of tens of meters (Maki et al., 2008). To realize the full capability of such models, rainfall estimates of commensurate resolution are needed. Additionally, beam broadening can also affect the quality of polarimetric radar measurements in the presence of non-uniform beam filling (Ryzhkov, 2007; Zhang and Qi, 2010). At long radar ranges, the radar beam extends a wider distance across beam, such that large gradients are more likely presented, which can result in substantial biases. One of the motivations of a networked sensing system is to achieve good cross-beam resolution using small range radars. In the current operational national network NEXRAD, each radar node probes the storm up to $460 \mathrm{~km}$, or $300 \mathrm{~km}$ in super-resolution mode, with $1^{\circ}$ beam. This corresponds to a cross-beam distance larger than $5 \mathrm{~km}$ at $300 \mathrm{~km}$ range, virtually preventing reliable quantitative rainfall estimates because of storm gradients either in vertical or in horizontal direction. NEXRAD delivers QPE products in $4 \times 4 \mathrm{~km}$ grids. If the range is contained within $40 \mathrm{~km}$, the cross-beam distance will be scaled down to $700 \mathrm{~m}$ and QPE products can be generated in much smaller grids. An individual node of the CASA network has a maximum range of about $40 \mathrm{~km}$ with a beam width of approximately 1.8 degrees, resulting in the largest resolution of about $1250 \mathrm{~m}$. However, there is significant overlap between the nodes of the radar network. Therefore, one can choose the smaller-resolution sample in the overlap regions. In a fully deployed network, there will 
always be some overlap. A detailed analysis of the sampling volume (or voxel) resolution as a function of beam width, network topology and degree of overlap can be found in Junyent and Chandrasekar (2009).

\subsection{Temporal resolution}

High temporal resolution real-time rainfall product generation is required in order to track fast moving or evolving precipitation events to predict the threats at the same time scale in order to elicit quick response. The current NEXRAD observations are updated every $5 \sim 6 \mathrm{~min}$, collecting volume information that can be used for many applications, beyond QPE. For example, the volume information and vertical profile are used to compute nowcasting as well as storm growth/decay trend measurement. Because radar measures the instantaneous rainfall rate, coarse temporal update rate likely leaves substantial sampling gaps in radar QPE for the cases of fast moving storms. Small sampling interval will be particularly valuable in the extreme rain storms. It only takes tens of minutes for an urban basin to reach its peak discharge from dry condition. As flood monitoring pinpoints down to specific blocks, the temporal variability is even higher and a sampling at $5 \sim 6 \mathrm{~min}$ can miss the local peaks. For shortrange radar, flexible waveforms and agile scan strategy can be adopted. In a dense radar network, scan patterns can be coordinated in an automatic and adaptive manner in response to the detected weather phenomena. For example, a full volume scan can be performed when a widespread shallow storm is present, whereas a narrower sector scan can be performed at multiple elevation angles to observe the vertical structure when deep convective storms are present. The CASA scan strategy systems are described in Zink et al. (2010).

\subsection{Beam height}

Radar measurement and rainfall estimates will be closer to the ground if they are obtained at lower heights. The radar beam also needs to be contained below bright bands or melting layers and above the significant mainlobe clutter to avoid QPE biases (Zhang and Qi, 2010). The Earth's curvature and the blockage by the local terrain can even prevent the coverage of raining regions of interest. The lowest beam height depends on radar range and the lowest available elevation angle. At long range, the beam height becomes extremely high because of the Earth's curvature, e.g., nearly $5.3 \mathrm{~km}$ at $300 \mathrm{~km}$ range even at $0^{\circ}$ elevation angle. In addition, the current regulations limit the lowest elevation angle used by NEXRAD to $0.5^{\circ}$, that is, one-half the antenna beamwidth. For good radar QPE, we prefer to have the lowest beam height to be kept around $1 \mathrm{~km}$. We choose the lowest useful height free of clutter contamination. While it is useful to choose the lowest height, one should be careful to avoid clutter contamination. Figure 1 shows the beam height as a function of the range from the radar, for short ranges using the $4 / 3$ Earth curvature model. It can be seen from Fig. 1 that $1 \mathrm{~km}$ height observation can be achieved at $1^{\circ}$ elevation angle if the maximum range is limited to $50 \mathrm{~km}$.

\subsection{Sensitivity}

The sensitivity of weather radar is directly proportional to the transmit power and inversely related to the square of radar range. Long duration light rain is not an apparent threat to an urban watershed, because the drainage can discharge the runoff in time. When using gauge measurements to validate radar QPE, the gauge may not respond well to light rain either. For example, a tip bucket rain gauge of $0.254 \mathrm{~mm}$ per bucket will not tip in a single 5-min interval for $2.5 \mathrm{~mm} \mathrm{hr}^{-1}$ rainfall, which is approximately translated to $30 \mathrm{dBZ}$ for radar reflectivity or $0.1 \mathrm{deg} \mathrm{km}^{-1}$ for the specific differential propagation phase. However, for X-band radar systems, an extra margin needs to be allocated for the transmit power budget to compensate for the rain attenuation. Statistically, rain attenuation is more likely to be higher at longer propagation paths. Altogether, short-range radar largely relaxes the constraint on sensitivity. Extensive studies have been conducted for rain attenuation margin needed for communication systems at higher elevation angles. However, data at very low elevations such as zero and few degrees are rare. Chandrasekar et al. (2009) have shown that, for the South Central US such as Oklahoma and Texas area, the attenuation margin needed for X-band radars is about $12 \mathrm{~dB}$. This margin will change with different climatic region. For example, it is likely to be smaller for the western coast of the US.

\subsection{Clutter mitigation}

The radar reflectivity for clutter echo can be written as

$Z_{\mathrm{ec}}<\mathrm{dBZ}>$

$=2 f(\alpha)+\sigma^{0}<\mathrm{dB}>+C+10 \log _{10} \frac{A_{c}<\mathrm{km}^{2}>}{V_{c}<\mathrm{km}^{3}>}$

where $f(\alpha)$ is the sidelobe pattern at clutter incidence angle, $\sigma^{0}$ is surface backscattering coefficient, $C$ is the radar constant, $A_{c}$ is the beam illuminated area, and $V_{c}$ is the effective radar resolution volume. At low grazing angles, $A_{c}$ is proportional to range. At low elevation angles and close ranges, ground-based radar can suffer significant ground clutter contamination. Reflection from buildings can have a high grazing angle, and in this case $\sigma^{0}$ can be larger by several orders. When these buildings intercept mainlobe, substantial beam blockage is produced. Use of the small-size radar enables flexible deployment if necessary, such that mainlobe clutter can be avoided. For example, rooftop installation on tall buildings can be afforded in an urban region. While main lobe clutter can be avoided by networked radar deployment and geometrical considerations, side lobe clutter is depended on the antenna beam characteristics as well as the prevailing clutter environment. CASA systems use a fairly advanced 


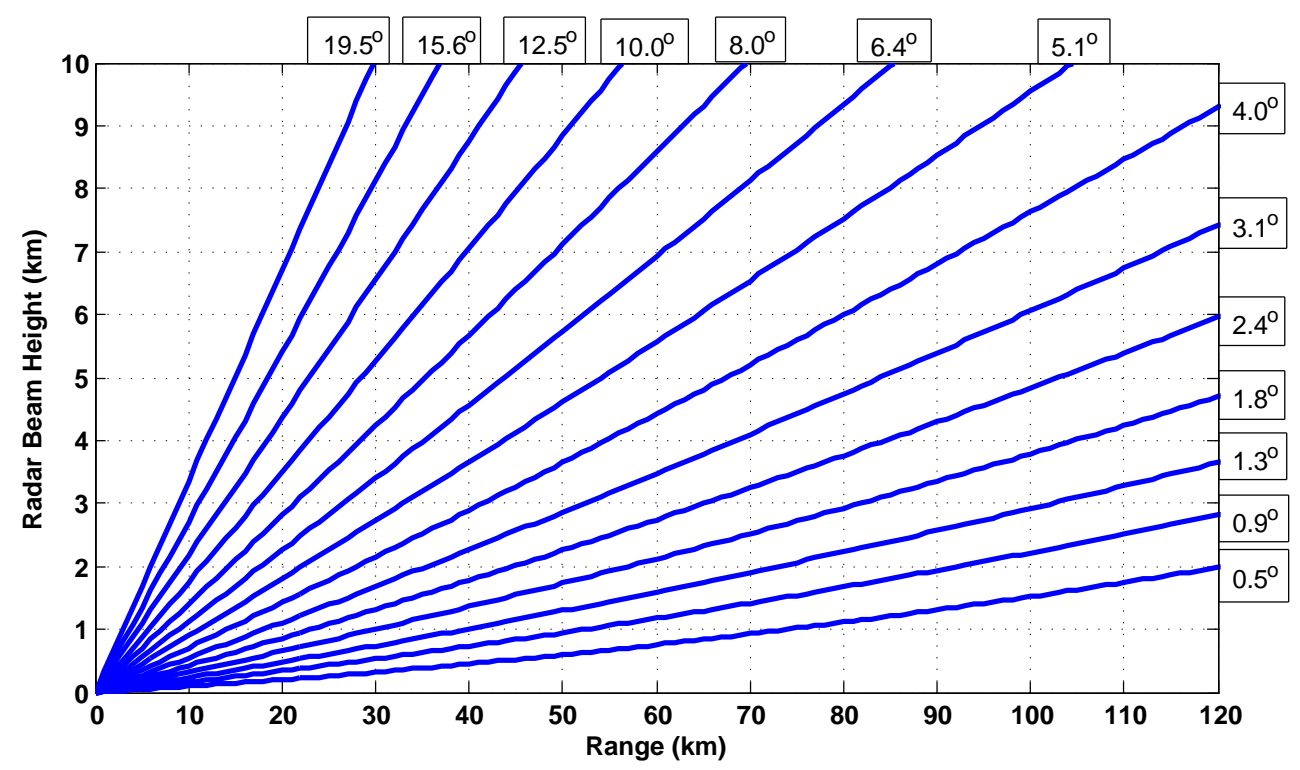

Fig. 1. Beam height vs. radar range at the elevation angles corresponding to one of the scan strategies of WSR-88D (VCP-12).

clutter filtering procedure that is very close to the GMAP technique (Siggia and Passarelli, 2004), except that it operates on the dual-polarized signals (Bharadwaj et al., 2010). It should be noted here that, since the main product used for rainfall is $K_{\mathrm{dp}}$, clutter filtering of dual polarized measurements is important. With a combination of networked radar application and advanced clutter filtering, the impact of clutter on rainfall estimation is mitigated in the CASA QPE system.

\section{X-band dual-polarization radar networks}

A distributed collaborative adaptive sensing (DCAS) system dynamically utilizes networked short-range radar units to achieve high spatiotemporal resolution (Junyent and Chandrasekar, 2009). In DCAS networks, distributed short-range radars are not only densely deployed, but the resources are also dynamically and adaptively allocated according to the storm evolution and user needs, achieving higher resolution in both space and time. All the radar nodes are operated collaboratively to provide the sensing functionality for the entire network coverage. Overlapped radar coverage is systematically designed to provide rich information for fusion, and neighboring radars can be coordinated in real time to pinpoint the features of interest. As a dense deployment, a DCAS network consists of many radar nodes, demanding a cost-efficient development and installation of a single radar node. As a result, the radar operating is moved to shorter wavelength, such as X-band. In fact in some instances, teams are experimenting with even higher frequencies such as $\mathrm{Ku}$ band (Yoshikawa et al., 2010).

\subsection{CASA IP1}

CASA has deployed a networked X-band radar test bed, named Integrated Project 1 (IP1), in southwestern Oklahoma. The IP1 test bed is the first DCAS system of this kind developed and operated by CASA, primarily to monitor and respond to severe thunderstorms, heavy rainfall, and severe winds. The test bed covers a $7000 \mathrm{~km}^{2}$ region in southwestern Oklahoma that receives an average of 4 tornado warnings and 53 thunderstorm warnings per year. The radars are located under the NEXRAD coverage of the KFDR and KTLX radars, as shown in Fig. 2.

It is an end-to-end system of a network of four, lowpower, short-range, dual polarization, Doppler radar units. Each radar is operated with a maximum range of $40 \mathrm{~km}$ and a maximum elevation angle of $30^{\circ}$. The four radar nodes are located in the towns of Chickasha (KSAO), Rush Springs (KRSP), Cyril (KCYR) and Lawton (KLWE), OK, and each radar node is approximately $30 \mathrm{~km}$ (or less) away from the next unit. All the nodes are connected to the Internet, and the IP1 radars transfer real-time observations to a central server known as System Operations Control Center (SOCC), located at a central facility, where radar data are fused and processed. A Meteorological Command and Control (MC\&C) system is also installed in SOCC which detects the weather features such as storm cells and circulations, optimizes resource allocation according to end-user's needs, and generates new scan tasks to reconfigure all the radar nodes (Zink et al., 2010). The architecture of the CASA system is shown Fig. 3. It shows the fundamental closed-loop aspect of the CASA system where in real time the scans are set according to the prevailing precipitation environment. This is a fundamental change from the current data push paradigm used in 


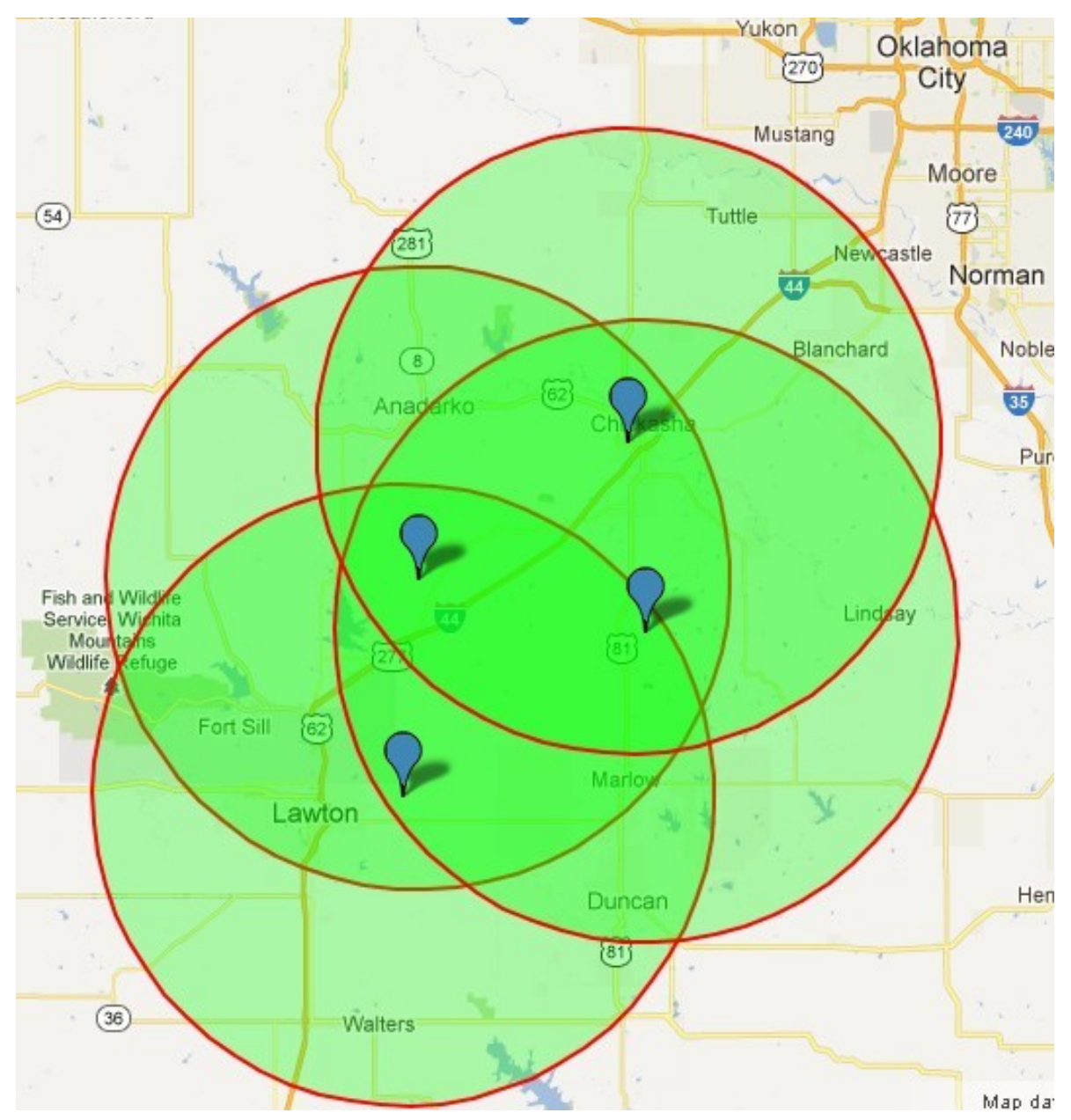

Fig. 2. The layout and location of the IP1 weather radar network. The coverage circles of IP1 radar are in radius of $40 \mathrm{~km}$.

weather radar systems. The system update time can be as fast as $30 \mathrm{~s}$ to $1 \mathrm{~min}$ due to the implementation of adaptive, coordinated sector scanning strategy.

\section{Dual polarization quantitative precipitation estimation}

Derivation of quantitative rainfall products from radar observations is known as a highly challenging process because of the inherent variability of hydrometeorological processes as well as the practical challenges of radar measurements in the context of rainfall estimation. Cifelli and Chandrasekar (2010) delineated these issues into two categories: physical science issues and engineering considerations. The microphysical variability can be largely accounted for using dual polarization measurements, such as combinations of the radar reflectivity, the differential reflectivity, and the specific differential propagation phase (Bringi and Chandrasekar, 2001). Rainfall estimation from $K_{\mathrm{dp}}$ is particularly appealing in X-band radar networks, because (1) it avoids the uncertainty in attenuation correction; (2) $K_{\mathrm{dp}}$ is sensitive to light rainfall at short wavelengths; (3) as the range slope of differential phase, it does not need network calibration for the four radar nodes. Deploying the radars as a network addresses the engineering challenges faced in rainfall estimation such as beam height, resolution and clutter issues, whereas using the $K_{\mathrm{dp}}$-based product addresses the physical science issues as discussed in Cifelli and Chandrasekar (2010).

Fundamentally, radar QPE is built upon physical models of the rain medium relating rain microphysics and radar observables (Bringi and Chandrasekar, 2001). The $R-K_{\mathrm{dp}}$ relation can be expressed in a power law form as

$R=a K_{\mathrm{dp}}^{b}$.

Due to the frequency scaling, $K_{\mathrm{dp}}$ responds well to low rainfall rate at X-band (compared to S-band), such that $R-K_{\mathrm{dp}}$ conversion can be directly applied in light rain circumstances; on the other hand, $K_{\mathrm{dp}}$ can also exhibit a steeper slope within an intense rain cell. For the same rainfall rate, $K_{\mathrm{dp}}$ at X-band is higher than that at S-band. In light rain, $K_{\mathrm{dp}}$ 


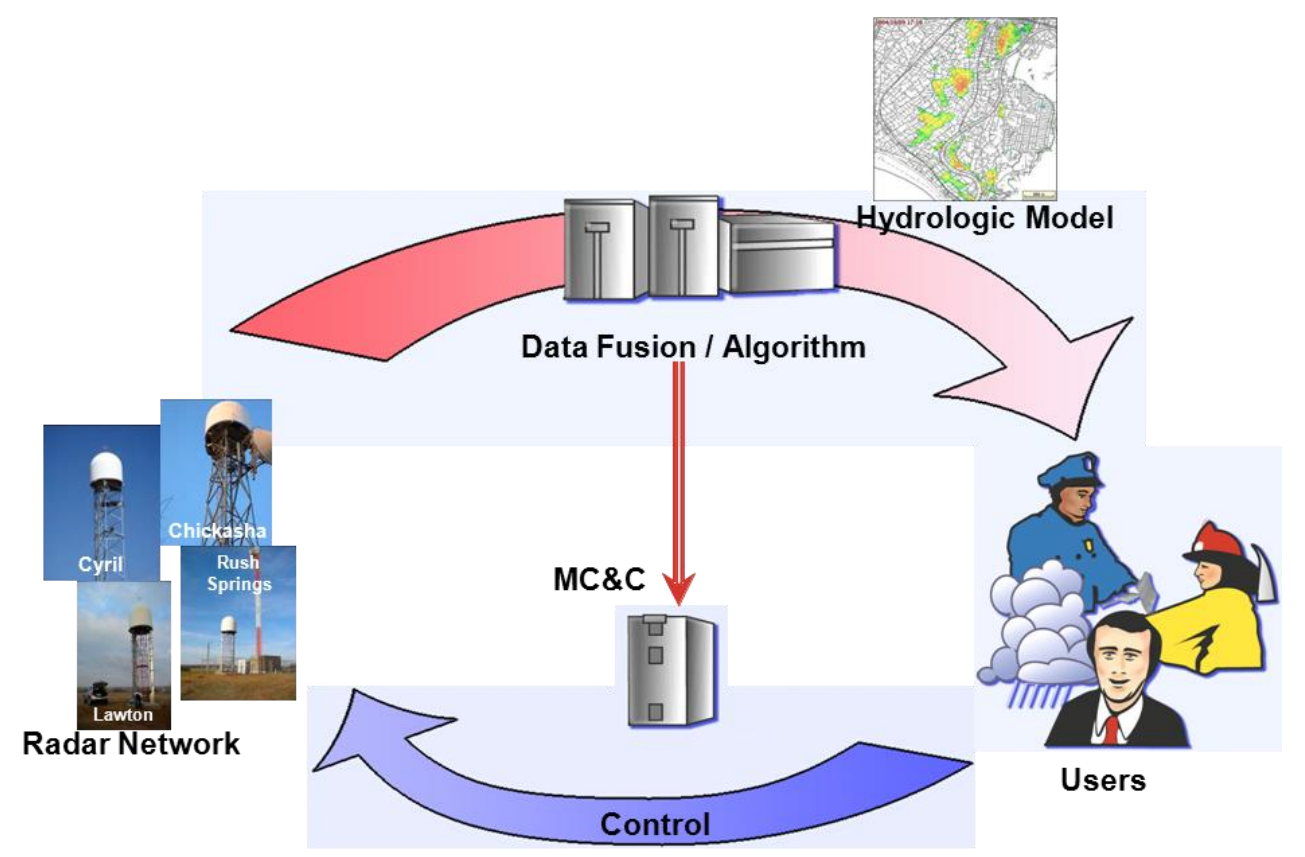

Fig. 3. CASA Architecture.

is small, and therefore it has to measure as the slope of $\Phi_{\mathrm{dp}}$. However, by going to higher frequencies, the $K_{\mathrm{dp}}$ threshold is smaller and hence $R\left(K_{\mathrm{dp}}\right)$ can be used for light rain. The estimation of $K_{\mathrm{dp}}$ is a challenge as the range derivative of the differential propagation phase profiles. It is subject to substantial fluctuations, especially at low rain rates, that need to be suppressed with a filter. However, the filter will smooth out the peaks on the other hand and introduce biases at high rain rates (Gorgucci et al., 1999). An adaptive $K_{\mathrm{dp}}$ algorithm automatically tuned to the spatial gradient of $K_{\mathrm{dp}}$ was implemented in the CASA QPE system that substantially reduces the fluctuation in light rain and the bias in heavy rain (Wang and Chandrasekar 2009). Figure 4a shows a snapshot of $K_{\mathrm{dp}}$ estimates for each IP1 radar. It can be seen that the $K_{\text {dp }}$ field is fairly stable in the low rain rate regions and is high in the storm cores.

The IP1 QPE test bed was designed with overlapping coverage among its radar nodes. The independence of $K_{\mathrm{dp}}$ on the radar calibration enables flexibility in combining the collocated $K_{\mathrm{dp}}$ estimates from all the radar nodes. It is the $K_{\mathrm{dp}}$ field, rather than the rainfall field, to be merged because we want to further reduce the variation on the input to the nonlinear $R-K_{\mathrm{dp}}$ conversion. The composite $K_{\mathrm{dp}}$ field comes from the radar with lowest beam height and nearest slant range, or from the radar with the best $K_{\mathrm{dp}}$ estimates. Moreover, the data availability is further enhanced by the overlapped topology in cases of heavy rainfall, or blockage or any other factor requiring redundancy demonstrating the operational strength of the network centric system.
In the IP1's DCAS scan strategy, the $2^{\circ}$ elevation scan is a surveillance scan, interleaved into a set of optimized scans from $1^{\circ}$ up to $30^{\circ}$ (Junyent et al., 2010). To drive the rainfall conversion, $K_{\mathrm{dp}}$ from the $2^{\circ}$ scan is used. At this angle, the clutter would come from sidelobes and its impact would be minimal after clutter filtering. Figure 4 a shows the estimated $K_{\mathrm{dp}}$ field for each IP1 radar within the system "heartbeat" of update time. The storm was passing over the center of network coverage, and each radar node had full or partial view of the storm. Figure $4 \mathrm{~b}$ displays the merged field based on the data quality of $K_{\mathrm{dp}}$ for rainfall application. The main consideration for using $K_{\mathrm{dp}}$ is choice of the closest range and lowest height for rainfall estimation (Wang and Chandrasekar, 2009). Alternate methods have been used to fuse the $K_{\mathrm{dp}}$ data from multiple radars, but there is not significant difference from one criterion over the other. The data fusion $K_{\text {dp }}$ is better than individual radar observations for rainfall applications. However, critically evaluating a large number of data sets, for practical considerations, a data quality-based fusion procedure is used. The major contributor to the data quality is the range because that corresponds to the lowest beam height. However, this may not be the best all the time. Since we are at X-band, the path attenuations from different directions are different. Sometimes the radar that has the best view is the one that has the least attenuation, if the signal values from the other radars are close to noise level. Thus, the data fusion of $K_{\mathrm{dp}}$ improves the estimate for rainfall applications. 

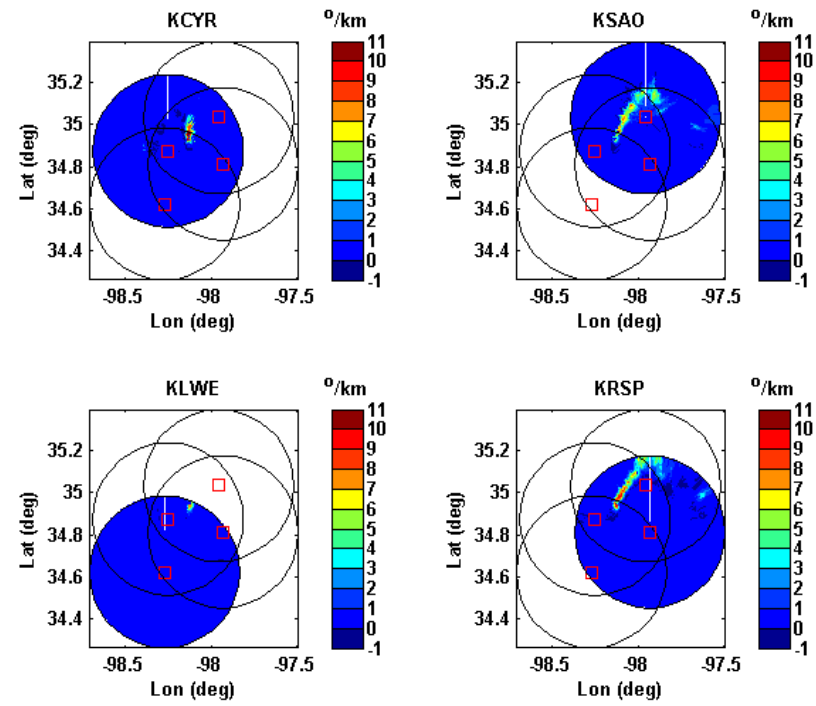

(a)

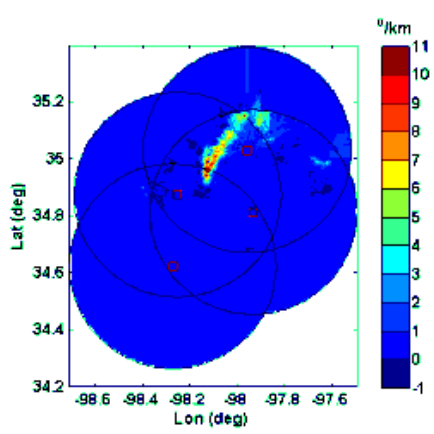

(b)

Fig. 4. Composite $K_{\text {dp }}$ estimates from the IP1 radar network, on 08:12 UTC, 27 May 2008. (a) the estimated $K_{\text {dp }}$ field for each IP1 radar, (b) the merged $K_{\mathrm{dp}}$ field based on the data quality of $K_{\mathrm{dp}}$.

\section{The QPE system functions and cross validation}

\subsection{QPE System functions}

The IP1 network is operated over a set of dynamic scan strategies where new scan protocols are continuously being developed and evaluated to serve the needs of a diverse enduser base, as shown in the architecture in Fig. 3. The network centric sensing can be implemented at varying time and spatial scales. The data flow architecture of QPE system is shown in Fig. 5a, demonstrating the data flow, whereas the functional system diagram for QPE product interface with end-users is shown in Fig. 5b. The high-resolution hydrologic model is eventually integrated into a DCAS network. As an end-to-end system, multiple aspects of flood forecasting prevention will be investigated, including data fusion,

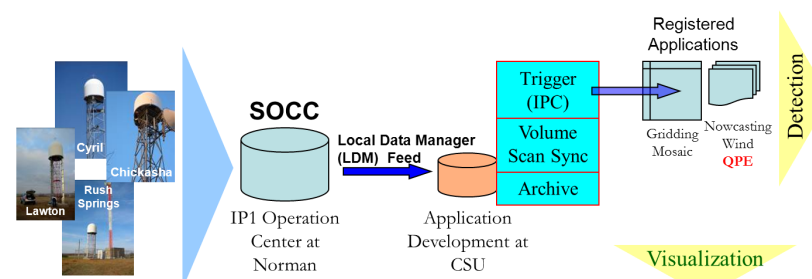

(a)

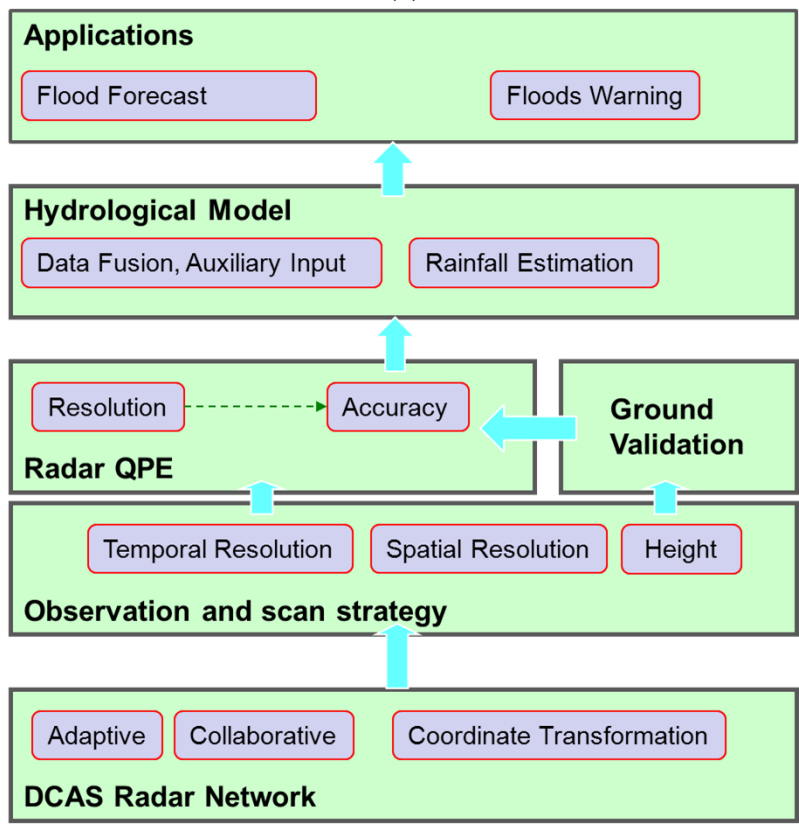

(b)

Fig. 5. (a) Data flow for QPE, (b) functional architecture of urban floods monitoring system integrated into a DCAS radar network.

rainfall estimation, short-term precipitation forecast, and user impact. The interaction between flood warnings and people response will also be investigated, in order to deliver better risk information to the users with different requirements. In addition to the standard QPE systems, an additional subsystem for nowcasting is also implemented in the QPE system (Ruzanski et al., 2011). This system has undergone extensive evaluation including the interface of QPE subsystem within the nowcasting system (Ruzanski and Chandrasekar, 2011).

In the QPE system, the $K_{\mathrm{dp}}$ estimates are produced on a node basis. This can be done physically either at the node level or at the central facility. The rainfall product generation happens at the central facility. There are several background processes that happen between the $K_{\mathrm{dp}}$ generation and rainfall estimation. Some of them include "data fusion" of rainfall estimation from multiple radar nodes. The governing principles for the QPE data fusion, just based on the radar nodes, are described in Sect. 4. This is the module that will be expended if external auxiliary data are brought in, such as a radar outside the network, or real time adjustment 
with in-situ sensors. One of the key processes in the central facility is the conversion of products from the radar coordinate system to the common geophysical coordinate system. Nearly all of the conventional radar products are generated with the radar as the origin. However, with more than one radar in the system, such a convention is not possible. The various products including the QPE, along with other products such as composite radar reflectivity factor, multipleDoppler wind velocity vectors, and nowcast products are produced in standard geophysical reference coordinates such as lat/long, Google Maps and GIS interface system that are used by the end-user community. In addition, these products are also delivered in a user-defined software environment such as, "Weather Scope", used by the Norman local emergency manager community, and WDSS II system, used by the local forecast community. There are special products that can be produced in addition to the standard rainfall maps (Surface Rainfall Intensity product), namely the hourly accumulation maps and single location rainfall accumulation trace, which can be used to compare against rain gauges.

\subsection{Cross validation}

CASA has conducted extensive validation studies on radar rainfall estimation using X-band dual-polarization radar. The metrics of validation include the mean bias, the normalized mean bias and the normalized standard error, defined respectively as follows:

$<e>=\left\langle R_{\mathrm{R}}-R_{\mathrm{G}}\right\rangle$

$<e>_{N}=\frac{\left\langle R_{\mathrm{R}}-R_{\mathrm{G}}\right\rangle}{\left\langle R_{\mathrm{G}}\right\rangle}$

$\mathrm{NSE}=\frac{\left\langle\left|R_{\mathrm{R}}-R_{\mathrm{G}}\right|\right\rangle}{\left\langle R_{\mathrm{G}}\right\rangle}$

where brackets denote sample average, $R_{\mathrm{R}}$ is the radar estimate, and $R_{\mathrm{G}}$ is the gauge measurement of instantaneous rainfall rate or hourly rainfall accumulation.

Since 2007, several intensive operation (IOP) experiments have been conducted in IP1, mostly during the spring storm season, to demonstrate and evaluate the fundamental concept of DCAS system and investigate the value added impact of this networked weather radar system compared to conventional weather radar systems (Chandrasekar et al., 2008). For use in the radar rainfall algorithm, a wide range of coefficients has been reported for the $R-K_{\mathrm{dp}}$ power law relation (Bringi and Chandrasekar, 2001) at both S-band and X-band. For fair comparison, the KOUN's $R-K_{\mathrm{dp}}$ relation was selected, which has been evaluated at S-band for the prototype WSR-88D system (Ryzhkov et al., 2005). The relation was scaled to X-band with respect to wavelength for rainfall conversion in the IP1 test bed, resulting in

$R=18.15 K_{\mathrm{dp}}^{0.791}$

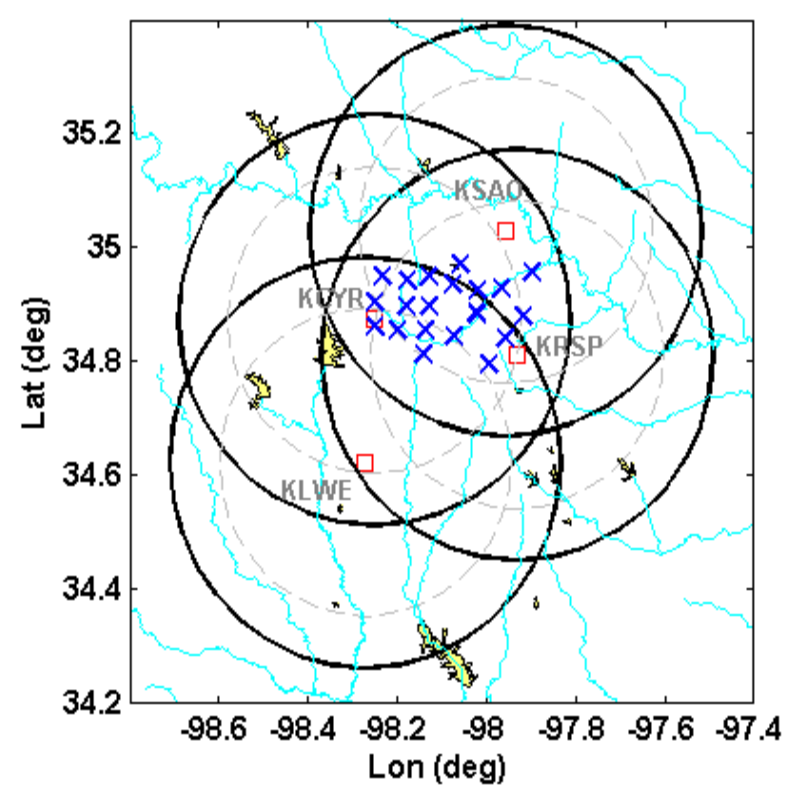

Fig. 6. Location of the USDA ground gauges in the CASA's IP1 network.

The performance of the IP1 QPE product was evaluated for all major rain events against the USDA Agriculture Research Service's gauge network (MicroNet) in the Little Washita watershed, which comprises 20 weather stations in the center of the test bed (as shown in Fig. 6).

In total, 42 storm events from the CASA experiments are analyzed in this paper, including events of different storm types such as severe thunderstorm, convective line, widespread stratiform rain, and cold front system. Overall, the hourly rainfall estimates compared to the gauge measurements have a very small bias of $3.74 \%$ and a normalized standard error of $25 \%$. These aggregate numbers show the excellent performance of the CASA QPE system over a 5-yr time period. The performance is also about a factor of three improvements over the current long-range weather radar estimates of rainfall reported in the literature.

\section{Sample products from the CASA QPE system}

The system architecture section described essentially three products from the CASA QPE system. They are namely, (a) instantaneous rainfall maps at high spatial and temporal resolution (500 $\mathrm{m}$ and $60 \mathrm{~s}$ ), (b) hourly rainfall accumulation maps produced every minute, and (c) point wise diagnostic traces of instantaneous rainfall rates and hourly accumulation. These products were chosen based on the feedback from the end-user community, primarily for flood forecast applications. It should be noted here that the hourly rainfall product is produced every minute to assist in estimating the flooding threat every minute. The extent of averaging can also be 


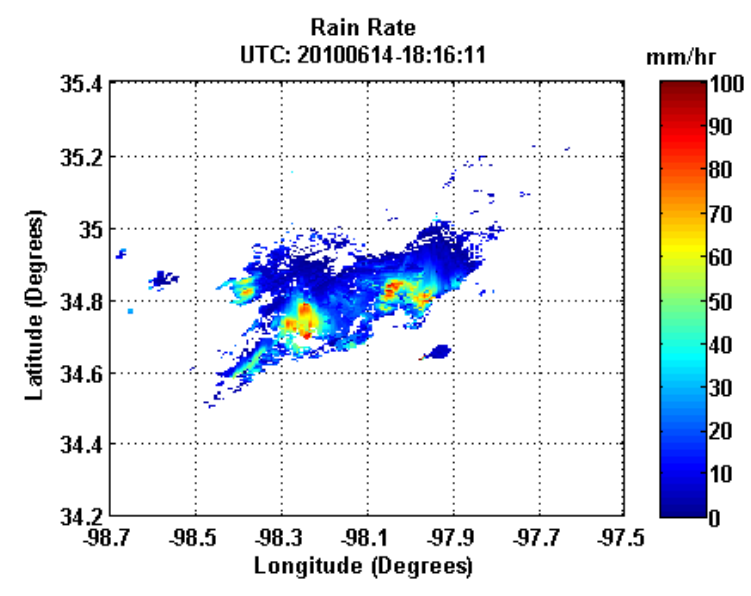

Fig. 7a. Instantaneous rainfall map.

adjusted to match the response scale of the basin of interest. For the IP-1 test bed, this time was set at $60 \mathrm{~min}$.

The following shows examples of instantaneous rainfall maps and hourly rainfall accumulation maps at the test bed, for 14 June 2010 case. Figure 7a shows a sample of instantaneous rainfall maps over a storm event, and the Fig. $7 \mathrm{~b}$ shows the hourly accumulation maps. In addition, point-wise traces are used for diagnostic purposes for comparison against gauges, and a detailed comparison against gauges at some of the Little Washita gauge network locations is shown in Fig. $7 \mathrm{c}$ for this rainfall event (at gauge number 154 location: Lat: 34.8553, Long: -98.1369). It can be clearly seen that the QPE system in CASA produces stable products for use in downstream applications such as flood forecast and input to hydrologic models. In addition to the standard QPE products, these products are also being tested with additional subsystems such as nowcasting. The current nowcasting product produces future time forecasts of reflectivity maps at fixed lead times - namely $5 \mathrm{~min}$ to half an hour. However, experimental products are being developed to produce nowcasting products of rainfall maps directly and they are under evaluation (Ruzanski and Chandrasekar, 2011). The preliminary results of Ruzanski and Chandrasekar (2011) show that the performance of direct QPE nowcasting is superior to going through the route of reflectivity and converting to rainfall.

\section{Summary and conclusions}

This paper described the CASA QPE system, the architecture, and its performance. This paper also described the process of high-resolution quantitative precipitation estimation (QPE) from a X-band dual-polarization radar network, which is expected to improve flood warning. High spatiotemporal resolution is also one of the essential requirements for prevention of flash floods and urban floods. Urban development reduces the response time of urban watersheds to rainfall and increases the peak discharge volumes. Equipped

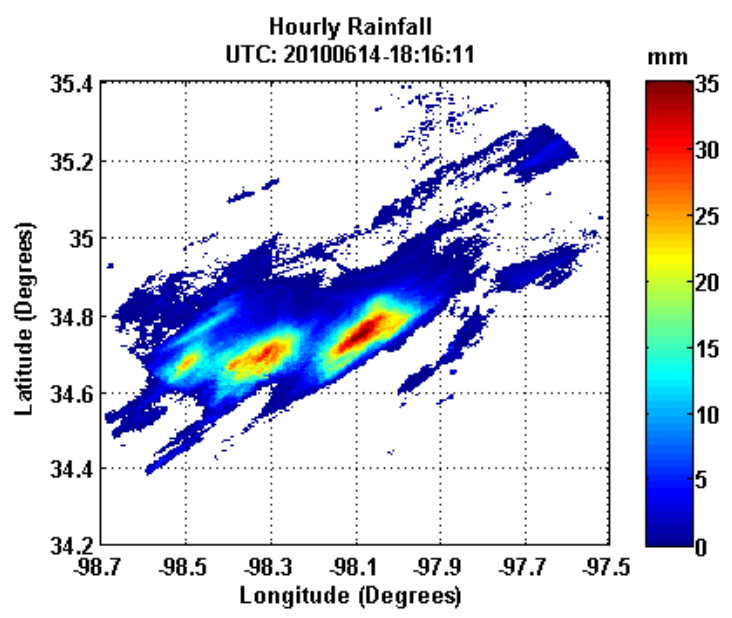

Fig. 7b. Hourly rainfall map.

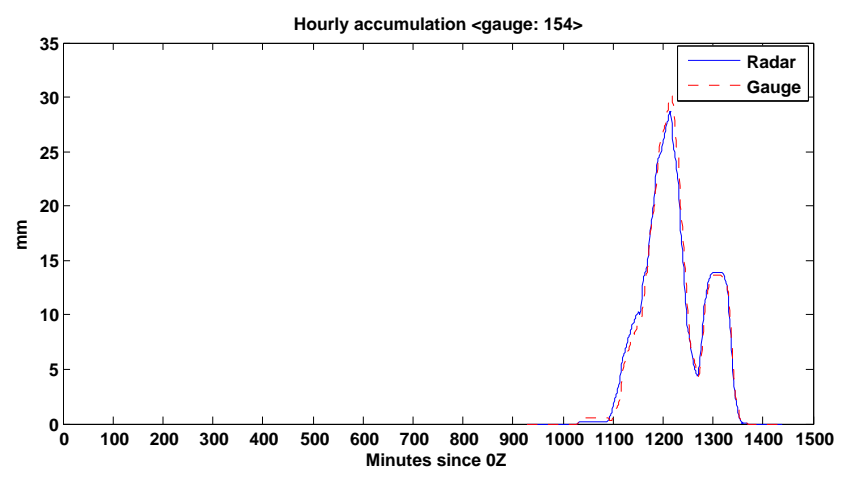

Fig. 7c. Radar and gauge hourly rainfall comparison at the location of gauge 154 for 14 June 2010 event.

with high-resolution rainfall maps, physically based distributed hydrologic models have significant advantage for accurate monitoring of urban watersheds and drainage networks.

A DCAS network of short-range radar can observe rain storms with high resolution and lower beam height closer to ground compared to current long- range radars. The current temporal resolution in CASA is $60 \mathrm{~s}$, which can be improved to $30 \mathrm{~s}$ for rainfall mapping. The spatial resolution is variable with a median value of about $300 \mathrm{~m}$ in the common area of the IP1 test bed. The performance of the CASA QPE system was demonstrated using networked radar rainfall mapping algorithm through ground validation using a rain gauge network. The fundamental limitations of long-range radar, such as beam broadening and higher altitude observations due Earth curvature blockage, are overcome by the dense deployment of a DCAS radar network. High- resolution observations can be achieved with smaller-size radar units throughout the network coverage using coordinated, collaborative scanning. Such a network also has great potential to serve as the complementary observing tool of NEXRAD, proving gap-filling data with improved temporal and spatial resolution. From a practical viewpoint, preparing the 
infrastructure to support the conventional long-range radar system is a major task in an urban environment, due to the social footprint of such systems. This is one of the major motivations driving the deployment of small-size X-band radars.

The QPE validation studies over $5 \mathrm{yr}$ have shown very good match between radar QPE and ground-based rain gauge network measurements, compared to the current state of the art. The excellent performance also shows the advantage of the network concept. The same studies also demonstrated the operational feasibility using X-band dual-polarization radar for accurate rainfall mapping and, in combination with the high resolution, the potential for driving the urban floods warning systems. Two types of products (namely instantaneous rainfall maps and rainfall accumulation maps) are shown to demonstrate the types of output products. In addition, point-wise trace of rainfall accumulation is used for validating against rain gauge networks. The interaction of the QPE subsystem with the nowcasting subsystem shows the potential for rainfall nowcasting.

Acknowledgements. This research is supported by the National Science Foundation via the NSF/Engineering Research Center Program ERC-0313747.

Edited by: N. Rebora

Reviewed by: H. Pohjola and one anonymous referee

\section{References}

Bringi, V. N. and Chandrasekar, V.: Polarimetric Doppler Weather Radar: Principles and applications, Cambridge University Press, 648 pp., 2001.

Bharadwaj, N., Chandrasekar, V., and Junyent, F.: Signal Processing System for the CASA Integrated Project I Radars, J. Atmos. Ocean. Technol., 27, 1440-1460, 2010.

Chandrasekar, V., McLaughlin, D. J., Brotzge, J., Zink, M., Philips, B., and Wang, Y.: Distributed Collaborative Adaptive Radar Network: Preliminary Results from the CASA IP1 Testbed, 2008 IEEE Radar Conference, Rome, Italy, 2008.

Chandrasekar, V., Willie, D., Wang, Y., Lim, S., and McLaughlin, D.: Attenuation margin requirements in a networked radar system for observation of precipitation, IEEE Int. Geoscience and Remote Sensing Symp., IGARSS 2009, 2, Cape Town, South Africa, IEEE, 957-959, 2009.

Cifelli, R., and Chandrasekar, V.: Dual polarization radar rainfall estimation. Rainfall: State of the Science, edited by: Testik, F. Y. and Gebremichael, M., Geophys. Monogr. Ser., 191, 105-125, AGU, Washington, DC, 2010.

Gorgucci, E., Scarchilli, G., and Chandrasekar, V.: Specific Differential Phase Estimation in the Presence of Nonuniform Rainfall Medium along the Path, J. Atmos. Ocean. Technol., 16, 16901697, 1999.

Junyent, F. and Chandrasekar, V.: Theory and characterization of weather radar networks, J. Atmos. Ocean. Technol., 26, 474-491, 2009.
Junyent, F., Chandrasekar, V., McLaughlin, D., Insanic, E., and Bharadwaj, N.: The CASA Integrated Project 1 Networked Radar System, J. Atmos. Ocean. Technol., 27, 61-78, 2010.

Maki, M., Maesaka, T., Misumi, R., Iwanami, K., Suzuki, S., Kato, A., Shimizu, S., Kieda, K., Yamada, T., Hirano, H., Kobayashi, F., Masuda, A., Moriya, T., Suzuki, Y., Takahori, A., Lee, D., Kim, D., Chandrasekar, V., and Wang, Y.: X-band Polarimetric Radar Network in the Tokyo Metropolitan Area - X-NET, The fifth European Conf. Radar Meteor. Hydrology, Helsinki, Finland, ERAD, 3.7., http://erad2008.fmi.fi/proceedings/extended/ erad2008-0020-extended.pdf., 2008.

McLaughlin, D. J., Chandrasekar, V., Droegemeier, K., Frasier, S., Kurose, J., Junyent, F., Philips, B., Cruz-Pol, S., and Colom, J.: Distributed Collaborative Adaptive Sensing (DCAS) for Improved Detection, Understanding, and Prediction of Atmospheric Hazards, 9th Symp. Integrated Obs. Assim. Systems, Amer. Meteor. Soc., 2005.

NRC: National Research Council: Flash Flood Forecasting Over Complex Terrain: With an Assessment of the Sulphur Mountain NEXRAD in Southern California, National Academy Press, 2005.

NRC: National Research Council: Observing Weather and Climate from the Ground Up: A Nationwide Network of Networks, National Academy Press, 2008.

Ruzanski, E. and Chandrasekar, V.: Scale filtering for improved nowcasting performance in a high-resolution X-band radar network IEEE Trans. Geosci. Remote Sens., 49, 2296-2307, 2011.

Ruzanski, E., Chandrasekar, V., and Wang, Y.: The CASA Nowcasting System, J. Atmos. Ocean. Technol., 28, 640-655, 2011.

Ryzhkov, A. V., Giangrande, S. E., and Schuur, T. J.: Rainfall Estimation with a Polarimetric Prototype of WSR-88D., J. Appl. Meteor., 44, 502-515, 2005.

Ryzhkov, A. V.: The Impact of Beam Broadening on the Quality of Radar Polarimetric Data., J. Atmos. Ocean. Technol., 24, 729744, 2007.

Saltikoff, E., Huuskonen, A., Hohti, H., Jarvinen, H., and Koistinen, J. : Quality assurance in the FMI Doppler weather radar network, Boreal Environ. Res., 15, 579-594, 2010.

Siggia, A. D. and Passarelli, R. E.: Gaussian model adaptive processing (GMAP) for improved ground clutter cancellation and moment calculation, Proc. of 3rd European Conf. on Radar in Meteorol. and Hydrology (CDROM), Visby, Sweden, 2004.

Wang, Y. and Chandrasekar, V.: Algorithm for Estimation of the Specific Differential Phase, J. Atmos. Ocean. Technol., 26, 2565-2578, 2009.

Yoshikawa, E., Ushio, T., Kawasaki, Z., Mega, T., Yoshida, S., Morimoto, T., Imai, K., and Nagayama, S.: Development and initial observation of high-resolution volume-scanning radar for meteorological application, IEEE Trans. Geosci. Remote Sens., 48, 3225-3235, 2010.

Zhang, J. and Qi, Y. : A Real-Time Algorithm for the Correction of Brightband Effects in Radar-Derived QPE, J. Hydrometeor., 11, 1157-1171, 2010.

Zink, M., Lyons, E. Westbrook, D., Kurose, J., and Pepyne, D.: Closed-loop Architecture for Distributed Collaborative Adaptive Sensing of the Atmosphere: Meteorological Command \& Control, International J. Sensor Networks, 7, No.1/2, 4-18, 2010. 